\title{
Investigation of Channel Interactions in a Nested Hall Thruster Part II: Probes and Performance
}

\author{
Sarah E. Cusson, ${ }^{*}$ Ethan T. Dale ${ }^{\dagger}$ and Alec D. Gallimore ${ }^{\ddagger}$ \\ University of Michigan, Ann Arbor, MI, 48109, USA
}

\begin{abstract}
Divergence angle and thrust measurements for a nested Hall thruster in different operating modes are presented. The X2 thruster, a two-channel nested Hall thruster, was run in both single channel and dual-channel modes. The divergence angle for each channel was measured in both modes using a near-field Faraday probe. In single channel mode, pressure was controlled via downstream injection as well as by flowing through the non-operating channel. Results show that thrust increases $5 \%$ and $11 \%$ in dual channel mode versus the summation of single-channels for high and low power operation respectively. When pressure is controlled via channel injection, thrust numbers for dual channel operation and the summation of single channel operation match. Beam current for the inner channel increases in dual channel mode and divergence angle decreases in dual channel mode. These results indicate that this increase in thrust is due to neutral ingestion from the adjacent channel and beam divergence decreases during dual channel mode.
\end{abstract}

\section{Nomenclature}

$\begin{array}{ll}()_{a} & =\text { anode } \\ ()_{\text {inner }} & =\text { inner channel } \\ ()_{\text {outer }} & =\text { outer channel } \\ d_{\text {OC }} & =\text { outer channel diameter } \\ F_{\text {th,coldgas }} & =\text { Cold gas thrust } \\ g & =\text { acceleration due to gravity } \\ I_{b} & =\text { beam current } \\ I_{s p} & =\text { specific impulse } \\ k & =\text { Boltzmann's constant } \\ \dot{m} & =\text { mass flow rate } \\ m_{X e} & =\text { discharge power } \\ P_{d} & =\text { thrust } \\ r & =\text { Gas temperature } \\ T & =\text { thermal velocity } \\ T_{g a s} & =\text { axial distance } \\ v_{t h} & =\text { efficiency } \\ z & =\text { divergence angle } \\ \eta & =\text { azimuthal angle } \\ \theta & \text { mass flow fraction } \\ \phi & \\ \chi & \end{array}$

*Ph.D. Candidate, Department of Aerospace Engineering, AIAA Student Member

${ }^{\dagger}$ Ph.D. Candidate, Department of Aerospace Engineering, AIAA Student Member

${ }^{\ddagger}$ Robert J. Vlasic Dean of Engineering, the Richard F. and Eleanor A. Towner Professor of Engineering, and an Arthur F. Thurnau Professor of Aerospace Engineering, and AIAA Fellow 


\section{Introduction}

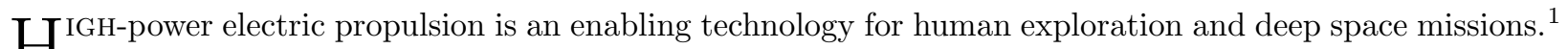
H However, there are many hurdles for the technology before it can be utilized on such missions. In particular, the main challenges to be solved are increasing the performance of low-power thrusters and advancing nested Hall thrusters to higher powers. ${ }^{1}$ Nested Hall thrusters concentrically nest multiple discharge channels to increase power while maintaining a small footprint. Nested Hall thrusters offer many advantages over single-channel thrusters, including an increased power-to-mass ratio and a larger performance range. Because these devices can be run in any combination of channels, it would allow a spacecraft to run a single-channel at low power for high efficiency or all channels at high power for high thrust. The X3, a three-channel nested Hall thruster, has a theoretical operational envelope from 2 to $200 \mathrm{~kW}$, a far greater range than any single-channel Hall thruster. ${ }^{2}$ Additionally, nested Hall thrusters are founded on a mature technology and thus are promising for near-term use on high-power electric propulsion missions.

In order to develop this technology such that it can be applied to critical space missions, the fundamental physics behind these devices must be well understood. Although there are still gaps in the understanding of single-channel thrusters, the increased complexity and novelty of nested Hall thrusters makes these gaps much more prevalent. For instance, multi-channel operation is one of the defining features of nested Hall thrusters but it is unclear how the plasma from multiple discharge channels interacts when they are in such proximity. The interaction between channels and the effect this has on the plume and performance remains one of the biggest questions left unanswered regarding nested Hall thrusters.

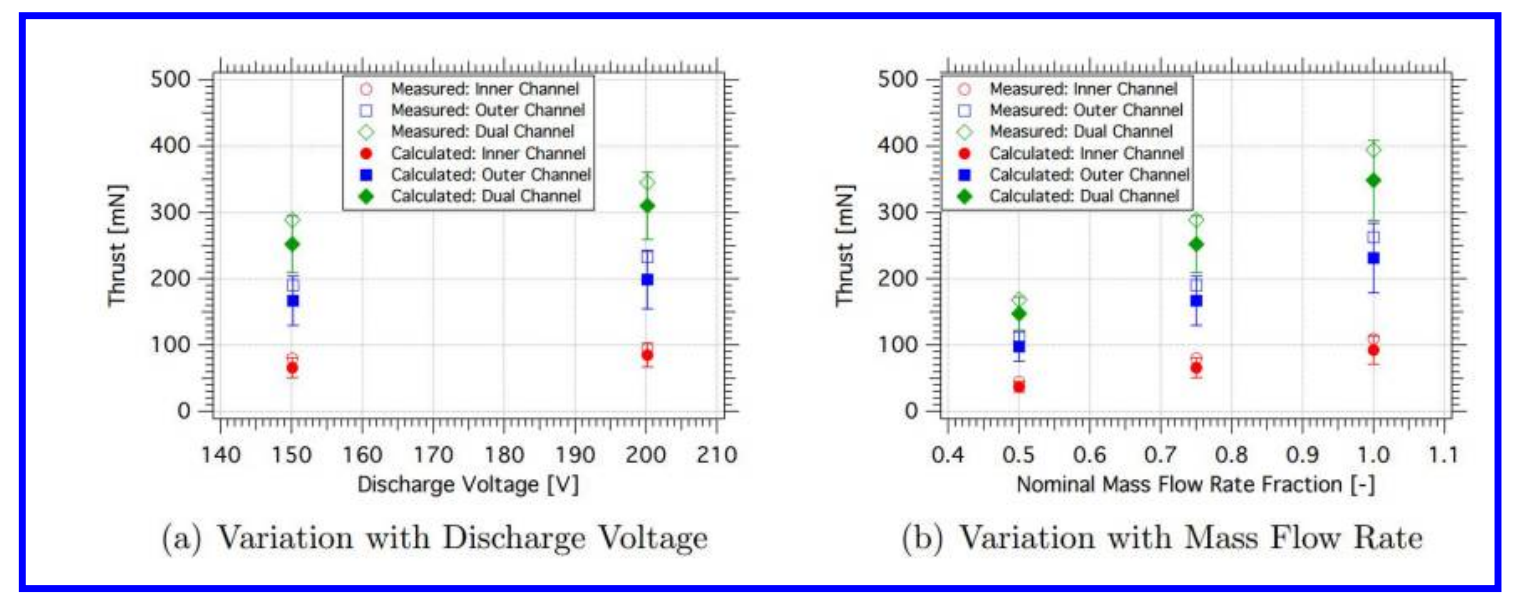

Figure 1: Comparison of thrust for single-channel mode versus dual channel operation shown by Liang ${ }^{3}$

Previous work done on nested Hall thrusters ${ }^{3,4}$ has revealed discrepancies in thrust measurements. Specifically, the thrust measured in multi-channel mode does not always equal the sum of the thrust of each channel operating alone. As seen in Figure 1, an increase in thrust was observed on the X2, a 6-kW two-channel thruster, in dual-channel mode versus the superposition of single-channel modes. The experiments were done with all operating conditions at the same chamber background pressure. Therefore, the increase is thrust was not deemed to be due to facility effects. ${ }^{3}$ However, the source of this increase in thrust was not investigated. The goal of the present work is to determine the mechanism behind the anomalous performance seen in nested Hall thrusters. The most likely source of this increased performance is neutral ingestion from the adjacent channel or a decrease in divergence angle leading to less cosine losses. A divergence angle decrease would suggest the acceleration region of the thruster has moved. This is discussed in the companion paper. ${ }^{5}$ To do this, performance and plume data for a nested Hall thruster was taken. Neutral gas was injected into the chamber to maintain a constant background pressure during all conditions. Gas was injected either downstream of the thruster or via the non-operating channel, which better simulates the local pressure during dual-channel operation. These conditions were used to determine whether neutral ingestion from the adjacent channel was the source of improved performance in dual-channel operation for the X2 nested Hall thrusters. Additionally, a pressure map near the thruster exit plane during cold gas flow was taken to see determine the local pressure near the thruster during operation. 


\section{Experimental Apparatus}

\section{A. Thruster}

The X2 is a two-channel nested Hall thruster jointly developed by the University of Michigan and the Air Force Research Laboratory. ${ }^{3,6,7}$ It was a proof-of-concept thruster and was the first nested Hall thruster developed in open literature. A picture of the thruster after fabrication and firing during this campaign can be seen in Figure 2. The thruster features two channels with similar designs and magnetic field topologies. ${ }^{7}$

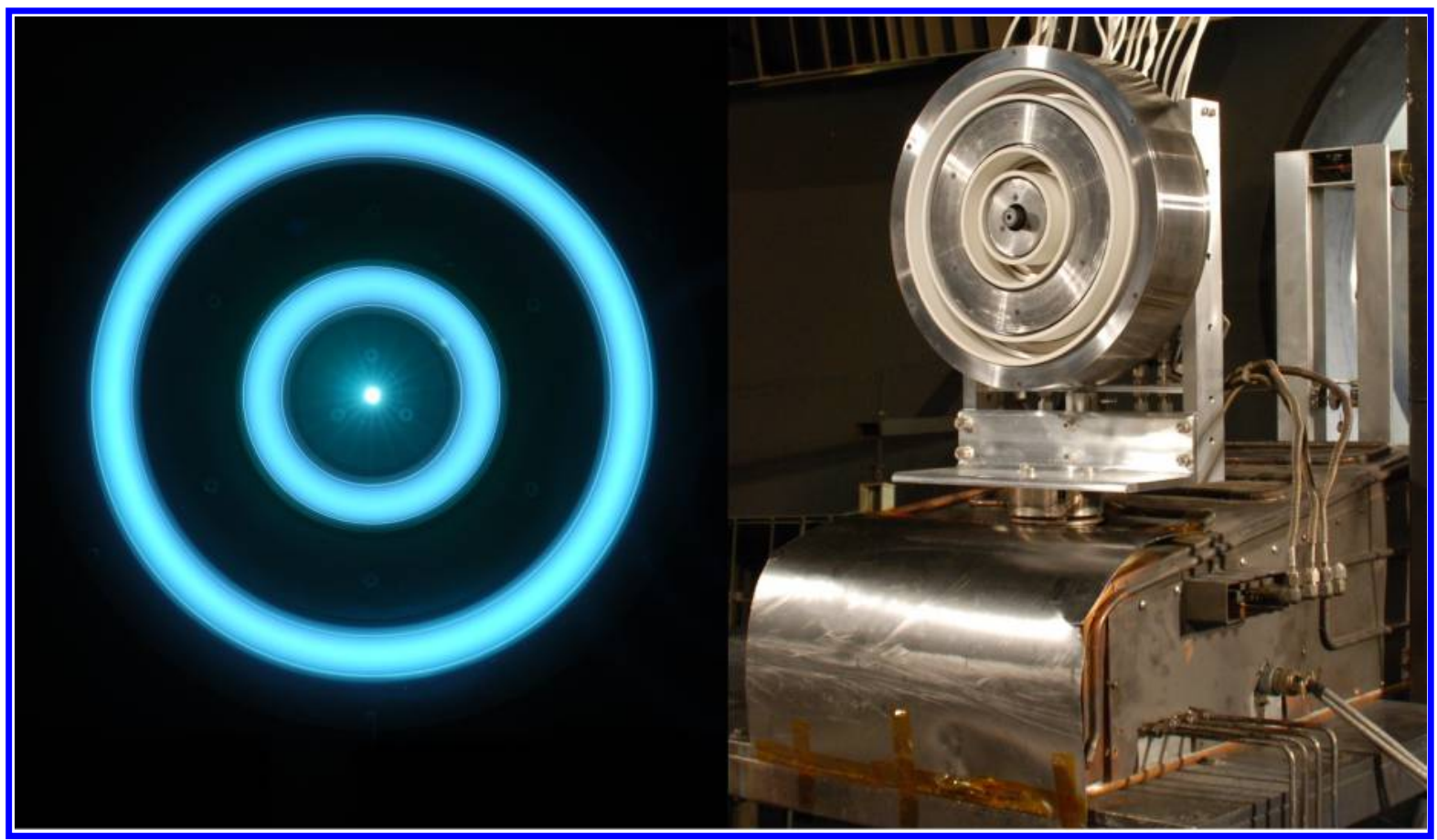

Figure 2: A picture of the $\mathrm{X} 2$, a 6 -kW nested Hall thruster ${ }^{7}$

Due to shared magnetic components, the inner channel magnetic field points radially inward while the outer channel field points radially outward. Each channel was operated with a separate power supply but the thruster was also designed to be able to use a single power supply. Power for the electromagnets, cathode heater, and cathode keeper were supplied via six commercially available power supplies. The thruster was supplied with research grade $(99.995 \%)$ xenon during operation via commercially available flow controllers. The thruster has a nominal operating power of 6 - $\mathrm{kW}$ and uses a single centrally-mounted LaB 6 cathode. During these experiments the anodes were biased to $150 \mathrm{~V}$ and the thruster body was grounded during operation.

\section{B. Thrust Stand}

An inverted-pendulum thrust stand, as described by Walker, ${ }^{8}$ was used to make thrust measurements. The thrust stand was run in null mode and multiple calibrations were done after thrust measurements to reduce uncertainty. Error analysis is performed as described by Polk. ${ }^{9}$ Efficiencies were then calculated using thrust data according to the following formulas:

$$
\begin{aligned}
\eta_{a} & =\frac{T^{2}}{2 \dot{m}_{a} P_{d}} \\
I_{s p, a} & =\frac{T}{\dot{m}_{a} g}
\end{aligned}
$$


Here $\eta_{a}$ is the anode efficiency, $T$ is the thrust in Newtons, $\dot{m}_{a}$ is the anode mass flow rate in kilograms per second, $P_{d}$ is the discharge power in Watts, $I_{s p, a}$ is the anode specific impulse in seconds and $g$ is the gravitational constant. Once thrust data was obtained, the thruster was moved onto a single-axis motion stage such that Faraday probe and laser-induced fluorescence studies could occur.

\section{Near-Field Faraday Probe}

In order to obtain divergence angle and beam current, a planar near-field Faraday probe was used. A picture of the probe can be seen in Figure 3. The design features a $3.2 \mathrm{~mm}$ tungsten collecting surface with a tungsten

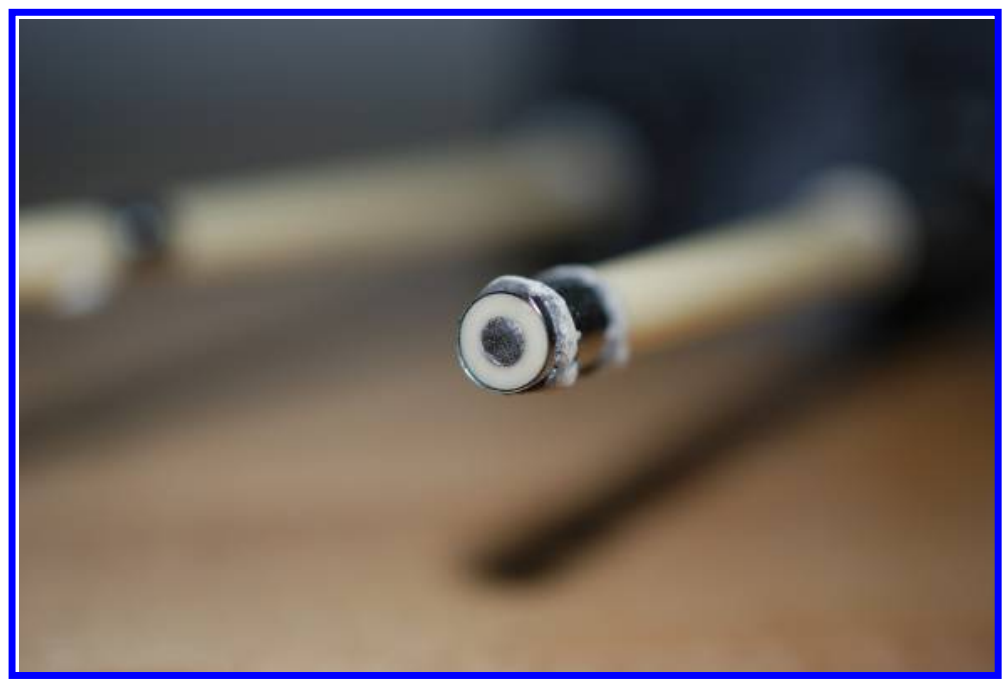

Figure 3: Near-field Faraday probe featuring a tungsten collector plate and guard ring

guard ring similar to that described by Hofer. ${ }^{10}$ The probe was biased to $-40 \mathrm{~V}$ during experiments to ensure ion saturation. Scans were taken at several axial locations by placing the probe on a two-dimensional motion stage system. During operation, the probe was constantly moving on a high-speed motion stage and data were collected using a Keithley 6485 Picoammeter at $60 \mathrm{~Hz}$. Divergence angle was calculated as described by Liang: ${ }^{3}$

$$
\theta(z)=\arctan \left(\frac{r_{2}-r_{\max }}{z}\right)
$$

Here $\theta$ is the divergence angle in degrees, $r$ is the radius integration limits in meters, and $z$ is the axial location in meters. Care must be taken when considering the integration limits. The method described by Reid, ${ }^{11}$ which uses a dynamic integration limit, is implemented. The two integration limits change with axial position and are defined as the location where the current density falls to $1 / e$ of the maximum. Due to plume merging in nested-channel thrusters, divergence angle measurements must be taken near-field while the plumes are still distinguishable from each other. After this point, only the outer channel divergence angle is obtainable. It is necessary to sample multiple axial locations until the axial position versus divergence angle trend has flattened. Once divergence angle becomes independent of location, this value can be used as the divergence angle of the thruster in that particular operating condition. The beam current is calculated as:

$$
I_{b}=\int_{0}^{2 \pi} \int_{r_{1}}^{r_{2}} j(r, z) r d r d \phi
$$

where $j$ is the current density and $\phi$ is the azimuthal angle around the thruster. The integration limits in equation 4 are chosen as the radial location where the current density fell to $10 \%$ of the maximum.

\section{Laser-Induced Fluorescence}

Time-averaged laser-induced fluorescence can be used to measure the acceleration zone location. A particular electronic transition in xenon is excited with a diode laser. Because ions are moving, they observe a Doppler 


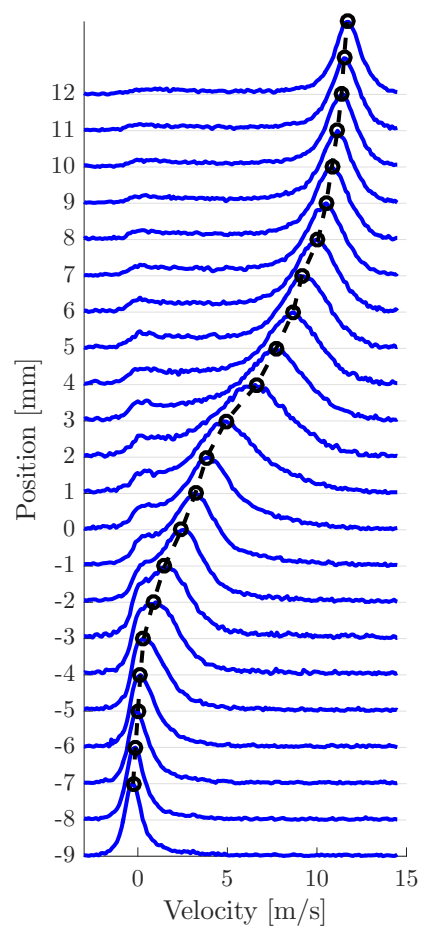

Figure 4: Example trace of the development of an LIF signal taken from the X2 thruster's outer channel in high power operation

shifted wavelength from the laser. Each wavelength corresponds to a particular velocity and by sweeping over a wide range of laser wavelengths, a velocity distribution can be inferred. ${ }^{12}$ By performing laser-induced fluorescence at regular intervals inside and outside the channel, the peak velocity can be found for each location. Figure 4 shows the velocity distribution at several axial positions inside the X2. ${ }^{12}$ Thus, the peak velocity can be plotted versus position to find the location of the acceleration region. More detail on both the technique and results can be found in this work's companion paper. ${ }^{5}$

\section{E. Facility}

All testing occurred in the Large Vacuum Test Facility (LVTF) at the University of Michigan. LVTF is a 9-meter long, 6-meter diameter vacuum chamber with a pumping speed of $240,000 \mathrm{~L} / \mathrm{s}$ on xenon using seven cryopumps. The thruster was run at two power levels, $4.5 \mathrm{~kW}$ and $2 \mathrm{~kW}$. The operational pressure during testing at high power was $6.8 \cdot 10^{-6}$ Torr-Xe and during testing at low power was $3.7 \cdot 10^{-6}$ Torr-Xe. Pressure measurements were taken with a Varian Series UHV-564 Ion Gauge located approximately three meters radially from the thruster and two meters downstream of the thruster. The background pressure was controlled via xenon gas injection. Xenon was injected downstream and away from the thruster during single-channel operation to match the background pressure seen during dual-channel operation. This ensures that background pressure was not a contributing factor to performance differences as per industry standards. ${ }^{13-16}$ Additionally, xenon was injected via the nonoperating channel during single-channel mode for the high-power case as seen in figure 5. This allowed for investigating the effect of local thruster pressure on channel interaction.

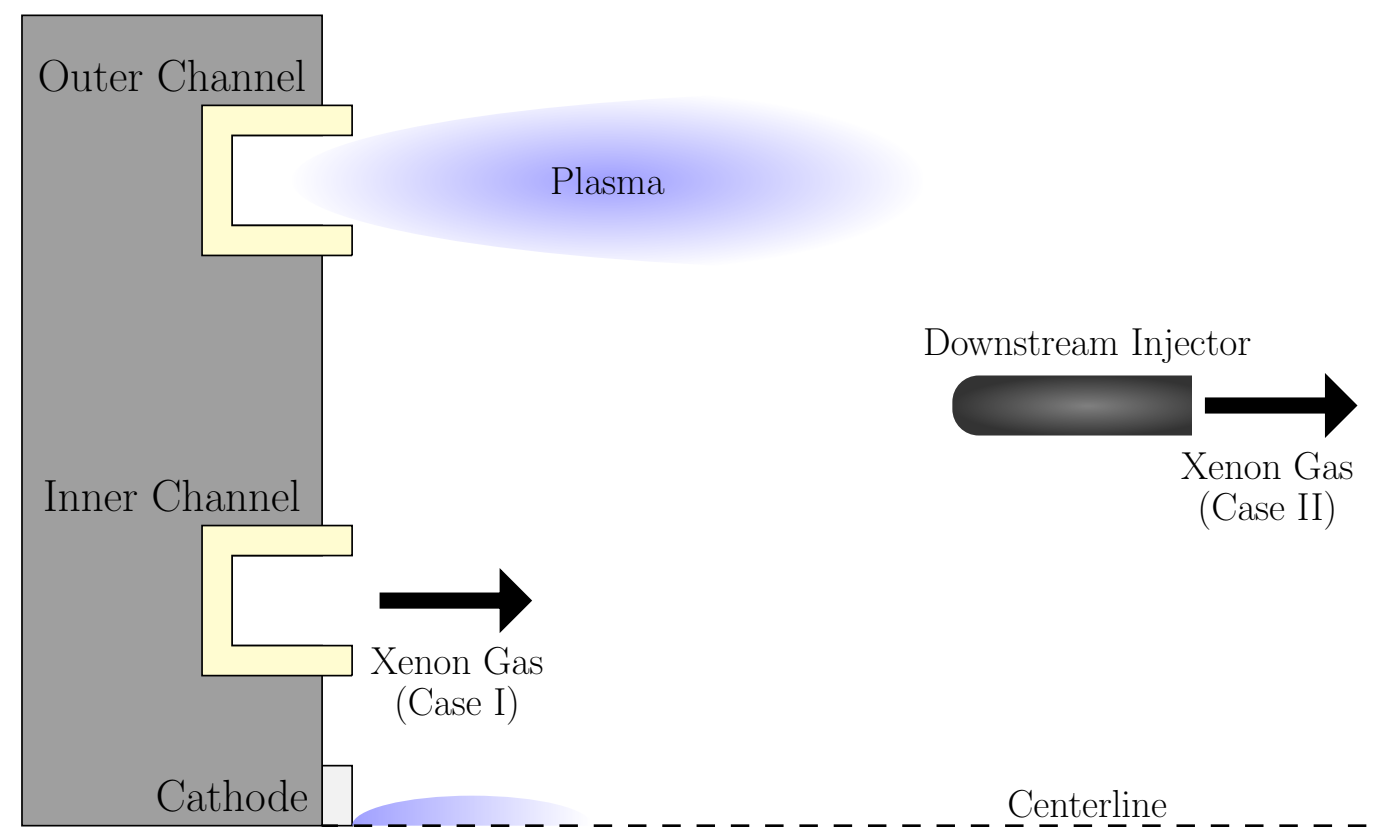

Figure 5: Neutral gas injection set-up for matching background pressure and investigating neutral ingestion

During these cases, the cathode flow fraction was kept constant for the operating channel at $10 \%$. The flow injected via the other channel was the nominal flow rate for that channel, and a small amount of gas was injected downstream to keep the total mass flow rate into the chamber constant. 


\section{F. Pressure Mapping}

In order to quantify the local pressure at the exit of the thruster, a Stabil Ion Gauge 370 was swept in front of the thruster while xenon was injected at nominal rates through the discharge channels and cathode. The setup for the test is seen in Figure 6 . The gauge was mounted with a gauge envelope similar to that recommended by

Dankanich. ${ }^{17}$ Pressure measurements were taken at various radial locations by moving the thruster on a single-axis motion stage. The entrance of the gauge envelop was location $10 \mathrm{~cm}$ downstream of the thruster exit plane. The mass flow rate during testing was the nominal mass flow rate for high-power operation which is $21.8 \mathrm{mg} / \mathrm{s}$ for the outer channel, $8.7 \mathrm{mg} / \mathrm{s}$ for the inner channel, and $3.0 \mathrm{mg} / \mathrm{s}$ for the cathode.

\section{G. Test Matrix}

The thruster was operated at eight different test points detailed in Table 1 . The discharge voltage for the thruster was 150 $\mathrm{V}$ for all conditions, and each channel used a separate power supply. Both channels of the thruster were run for 3 hours each (to-

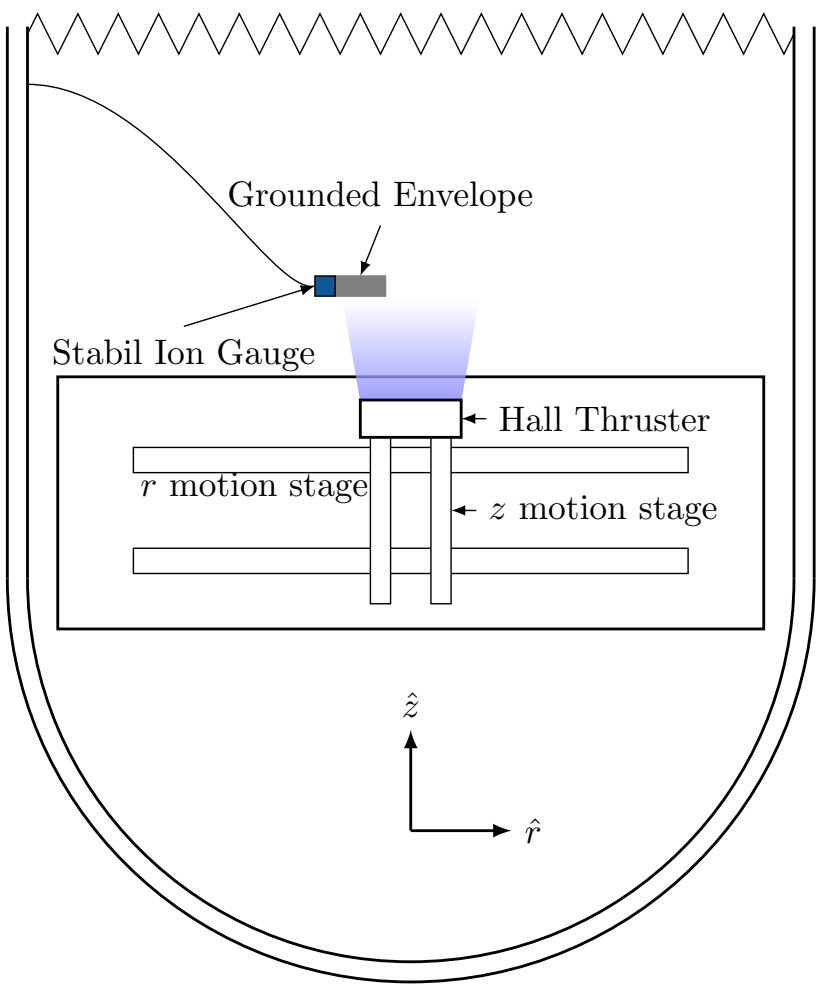

Figure 6: Experimental setup for pressure mapping of the near-field of the thruster using a Stabil Ion Gauge Series 370 gether or separately) after pumpdown and prior to any measurements being taken in order to ensure operating current was steady and unchanging. Additionally, this allowed the system to reach an equilibrium point as temperatures are rapidly changing during initial start-up and may affect any diagnostic measurements. Operating the thruster in all possible

Table 1: Summary of Test Points for the Experiment

\begin{tabular}{c|c|c|c|c} 
Test Point & $\begin{array}{c}\text { Inner Channel } \\
\text { Power }[\mathrm{kW}]\end{array}$ & $\begin{array}{c}\text { Outer Channel } \\
\text { Power }[\mathrm{kW}]\end{array}$ & $\begin{array}{c}\text { Total } \\
\text { Power }[\mathrm{kW}]\end{array}$ & $\begin{array}{c}\text { Gas Injection } \\
\text { Point }\end{array}$ \\
\hline 1 & 0.5 & 1.5 & 2 & N/A \\
\hline 2 & 0.5 & - & 0.5 & Downstream \\
\hline 3 & - & 1.5 & 1.5 & Downstream \\
\hline 4 & 1.25 & 3.25 & 4.5 & N/A \\
\hline 5 & 1.25 & - & 1.25 & Downstream \\
\hline 6 & 1.25 & - & 1.25 & Channel \\
\hline 7 & - & 3.25 & 3.25 & Downstream \\
\hline 8 & - & 3.25 & 3.25 & Channel
\end{tabular}

configurations at the same test point allows direct comparisons to be made between single-channel operation and nested-channel operation. For test points 1-3, the applied magnetic field to the thruster was identical for each case and the background pressure in the chamber was controlled to the same value for each point. The same is true for test points $4-8$, but the values are different than for points 1-3. Testing at two different power levels $(2 \mathrm{~kW}$ and $5 \mathrm{~kW}$ ) allowed for comparison between operating conditions to evaluate whether trends are case-specific or apply in general to the thruster. 


\section{Results and Discusson}

\section{A. Pressure Mapping}
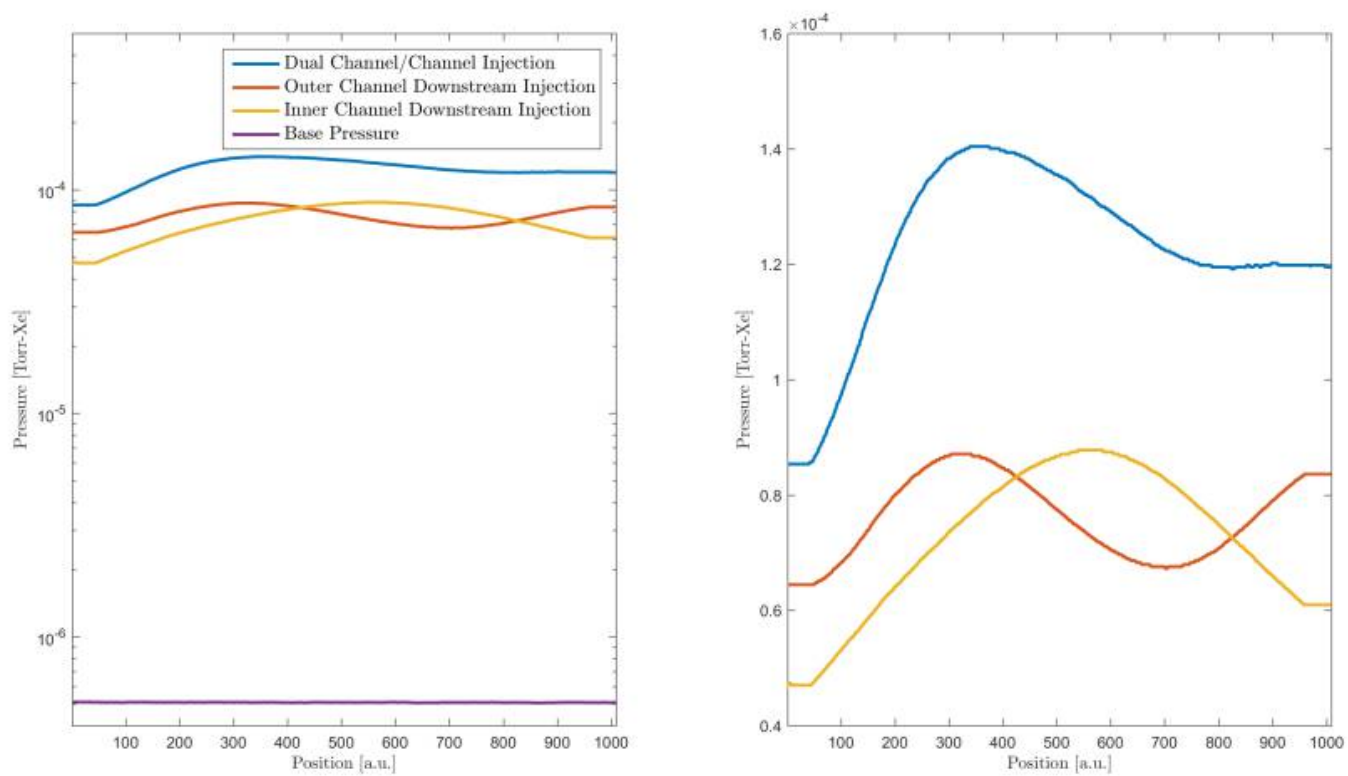

Figure 7: Neutral pressure as a function of radial location for the X2 at $0.35 d_{O C}$.

Results from the radial sweeping of a Stabil Ion Gauge in front of the thruster can be seen in Figure 7. The results show that with a base pressure of $5.1 \cdot 10^{-7}$ Torr-Xe, the maximum pressure in dual-channel mode at $0.35 d_{O C}$ from the exit plane is $1.4 \cdot 10^{-3}$ Torr-Xe while the maximum pressure with downstream injection is $8.7 \cdot 10^{-5}$ Torr-Xe for both the inner and outer channels. The pressure profile for dual-channel mode matches that for single-channel with channel injection because the same flow is being injected into the chamber in the same locations. While not explicitly shown on the graph, adding the outer channel and the inner channel profiles produces the same profile as dual-channel mode. However, because the total flow into the chamber when summing the two profiles is higher, the magnitude is higher. This data shows that neutral density near the thruster is much higher in dual channel mode than in single channel mode even when the background pressure is the same.

\section{B. Thrust}

The thrust measurements for the channel injection cases were corrected for cold gas thrust since simply flowing gas through the other channel produces some non-negligible thrust. The cold gas thrust was approximated and subtracted out from the total thrust numbers. The velocity of the particles exiting the channel were approximated as their thermal velocity $[\mathrm{m} / \mathrm{s}]$,

$$
v_{t h}=\sqrt{\frac{8 k T_{g a s}}{\pi m_{X e}}}
$$

where $\mathrm{k}$ is Boltzmann's constant, $T_{\text {gas }}$ is the gas temperature in Kelvin, and $m_{X e}$ is the mass of a xenon atom in kilograms. The temperature of the gas was assumed to be room temperature, or $300 \mathrm{~K}$. The cold gas thrust was then calculated as:

$$
F_{t h, \text { coldgas }}=\dot{m} v_{t h}
$$

where $\dot{m}$ is the mass flow through the channel that is not operating. The thrust due to cold gas flow for the inner channel was calculated to be $2 \mathrm{mN}$ while the cold gas thrust for the outer channel was calculated to be $5 \mathrm{mN}$. The thrust would increase with the square root of temperature however because the order of 
Table 2: Thrust Results for the Various Test Points and Injection Location for Reference

\begin{tabular}{c|c|c|c|c} 
Test Point & $\begin{array}{c}\text { Thrust } \\
{[\mathrm{mN}]}\end{array}$ & $\begin{array}{c}\text { Uncertainty } \\
{[\mathrm{mN}]}\end{array}$ & $\begin{array}{c}\text { Power } \\
{[\mathrm{kW}]}\end{array}$ & Injection Location \\
\hline \hline 1 & 141.090 & 4.919 & $1.90 \pm 0.06$ & N/A \\
\hline 2 & 31.701 & 3.176 & $0.47 \pm 0.02$ & Downstream \\
\hline 3 & 95.706 & 2.752 & $1.39 \pm 0.08$ & Downstream \\
\hline 4 & 357.753 & 4.552 & $4.46 \pm 0.08$ & N/A \\
\hline 5 & 92.736 & 2.718 & $1.26 \pm 0.05$ & Downstream \\
\hline 6 & 96.619 & 2.993 & $1.27 \pm 0.05$ & Outer Channel \\
\hline 7 & 248.073 & 3.324 & $3.26 \pm 0.08$ & Downstream \\
\hline 8 & 265.336 & 3.604 & $3.24 \pm 0.07$ & Inner Channel \\
\hline
\end{tabular}

magnitude is much lower than the actual thrust, the thrust due to cold gas flow is almost negligible even if gas heating occurs within the channel before expulsion.

The results shows that dual-channel mode produces more thrust than the superposition of single-channel

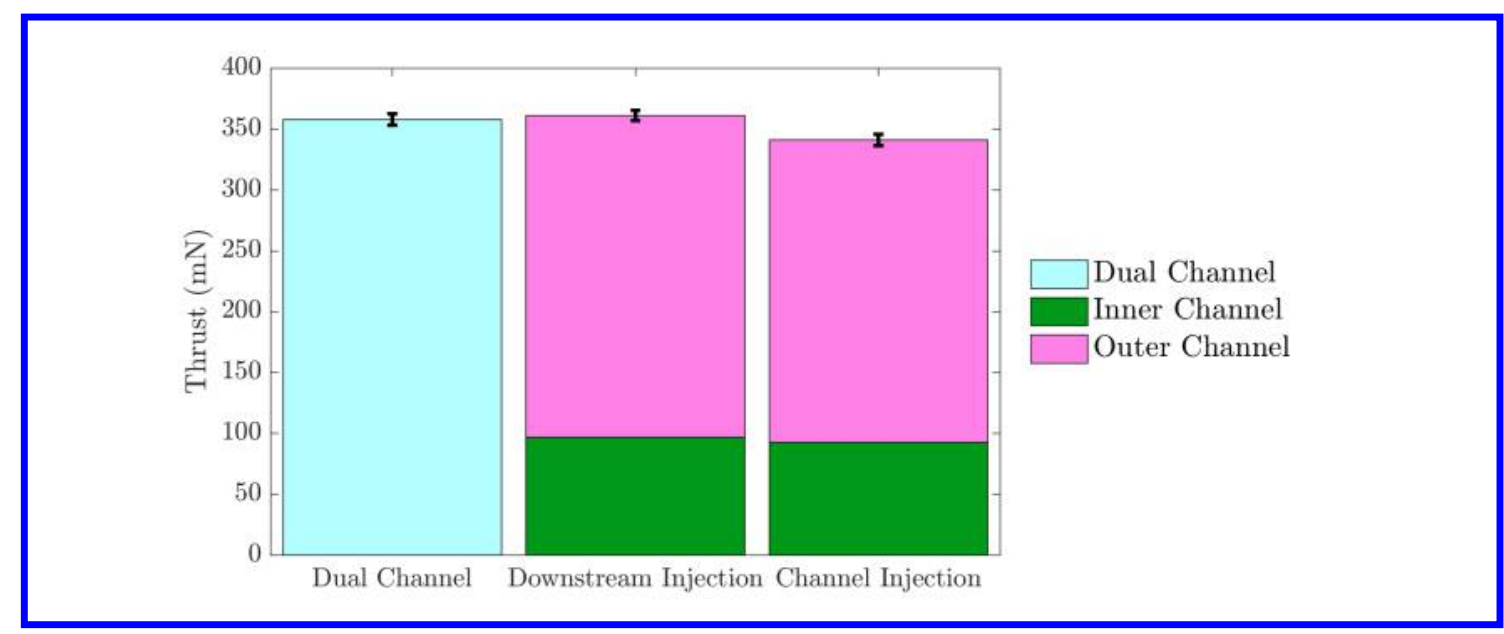

Figure 8: Comparison of dual channel thrust with summation of single channel thrusts for both injection cases.

modes when controlling pressure via downstream injection. The increase during high-power operation is $5 \%$ and the increase during low-power operation is $11 \%$. These results are consistent with what was seen by Liang. ${ }^{3}$

The thrust numbers indicate that the X2 does indeed have anomalously high performance in dual-channel mode. Interestingly, when the background pressure is controlled to the same value via channel injection the thrust is the same as in dual-channel mode as seen in Figure 8. This suggests that the presence of more neutrals near the discharge is leading to the increase in thrust. In other words, the increase in thrust is likely due to neutral ingestion caused by the increased local pressure during dual-channel mode.

Table 3 shows the $I_{s p}$ and $\eta_{a}$ for all test points. An important result comes from a comparison of cases 7 and 8, which shows that the efficiency and specific impulse for the outer channel increase when the pressure is matched via channel injection versus downstream injection. To further compare these results, it is necessary to get an effective dual-channel efficiency and specific impulse for the single-channel modes. This is because the inner and outer channels do not perform equally, and it is thus difficult to compare these cases to dual-channel mode. Therefore, each of the values for single-channel modes are weighted by their 
Table 3: Anode Efficiency and Specific Impulse for the Various Test Points

\begin{tabular}{c|c|c|c} 
Test Point & Anode Efficiency & $\mathrm{I}_{a, s p}[\mathrm{~s}]$ & Injection Location \\
\hline \hline 1 & $0.34 \pm 0.02$ & $940 \pm 31$ & N/A \\
\hline 2 & $0.24 \pm 0.05$ & $735 \pm 69$ & Downstream \\
\hline 3 & $0.30 \pm 0.02$ & $894 \pm 24$ & Downstream \\
\hline 4 & $0.47 \pm 0.01$ & $1196 \pm 14$ & N/A \\
\hline 5 & $0.39 \pm 0.02$ & $1086 \pm 29$ & Downstream \\
\hline 6 & $0.42 \pm 0.03$ & $1132 \pm 32$ & Outer Channel \\
\hline 7 & $0.44 \pm 0.01$ & $1163 \pm 14$ & Downstream \\
\hline 8 & $0.50 \pm 0.02$ & $1239 \pm 15$ & Inner Channel \\
\hline \multicolumn{3}{|c}{} & \\
\hline
\end{tabular}

mass flow fraction to calculate the effective efficiency, as follows:

$$
\begin{array}{r}
\eta_{\text {effective }}=\chi_{\text {inner }} \eta_{\text {inner }}+\chi_{\text {outer }} \eta_{\text {outer }} \\
I_{\text {sp }, \text { effective }}=\chi_{\text {inner }} I_{\text {sp }, \text { inner }}+\chi_{\text {outer }} I_{\text {sp }, \text { outer }} \\
\chi_{\text {inner }}=\frac{\dot{m}_{\text {inner }}}{\dot{m}_{a}} \quad \chi_{\text {outer }}=\frac{\dot{m}_{\text {outer }}}{\dot{m}_{a}}
\end{array}
$$

where $\eta_{\text {effective }}$ is the effective anode efficiency, $\chi$ is the mass flow fraction, ()$_{\text {inner }}$ refers to the inner channel, ()outer refers to the outer channel, and $I_{s p, e f f e c t i v e}$ is the effective anode specific impulse. Calculating these quantities yields the results in table 4. These results show that when pressure is controlled via

Table 4: Effective anode Efficiency and Specific Impulse for the Single Channel Modes versus Dual Channel Mode

\begin{tabular}{c|c|c} 
Test Point & (Effective) Anode Efficiency & (Effective) $\mathrm{I}_{s p, a}[\mathrm{~s}]$ \\
\hline \hline 4 & $0.47 \pm 0.01$ & $1196 \pm 14$ \\
\hline $5+7$ (Downstream) & $0.42 \pm 0.02$ & $1141 \pm 33$ \\
\hline $6+8$ (Channel) & $0.47 \pm 0.03$ & $1208 \pm 36$ \\
\hline
\end{tabular}

channel injection, the effective anode efficiency and specific impulse match that of dual-channel mode, while controlling via downstream injection yields effective an anode efficiency and specific impulse lower than the dual-channel values. This again indicates that the increased neutral density due to the adjacent discharge channel is producing an increase in performance. 


\section{Faraday Probe Measurements}

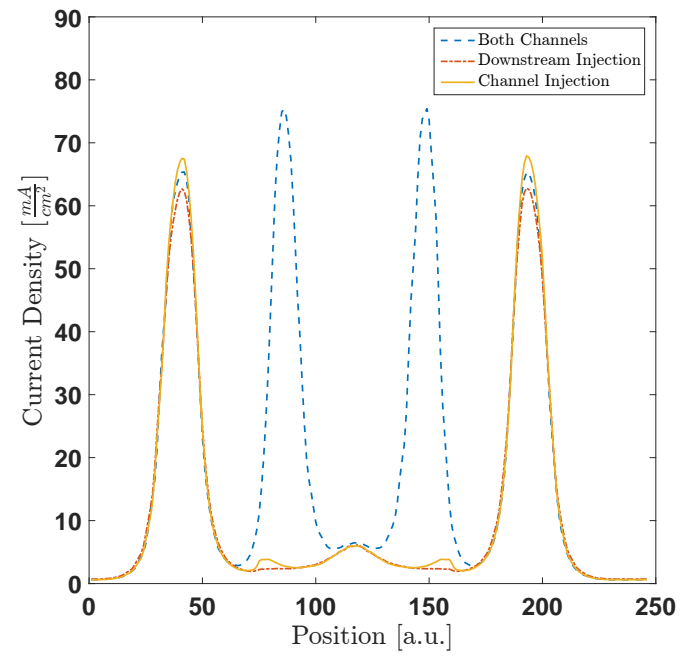

(a) High power operation.

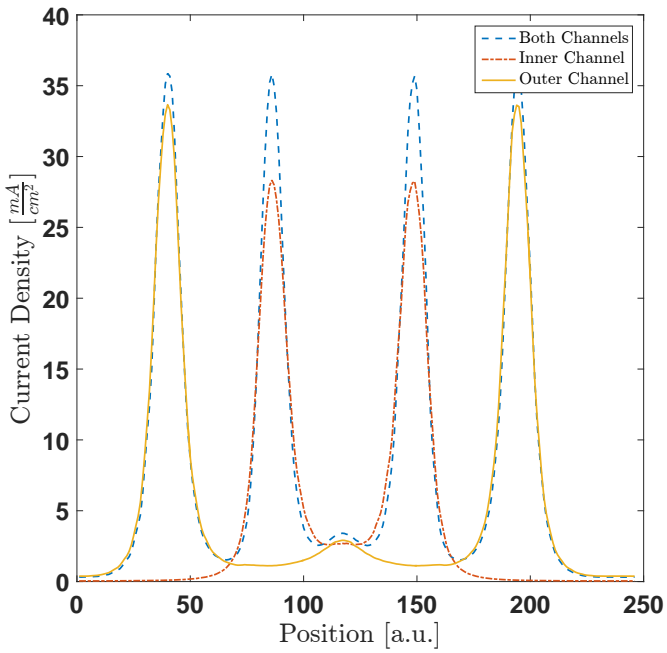

(b) Low power operation.

Figure 9: Example current density traces for all operating conditions taken at $0.17 d_{O C}$.

Near-field Faraday probe measurements were used to calculate the beam current and divergence angle. The divergence angle for each point was taken at two locations azimuthally around the thruster and then averaged together. Divergence angle as a function of axial location downstream of the thruster can be seen in Figure 11 and 10. Figures 10b, 11a, and 11b show that for all axial locations except one, the divergence angle in dual-channel mode is lower than the divergence angle in single-channel mode. This is because, as seen in

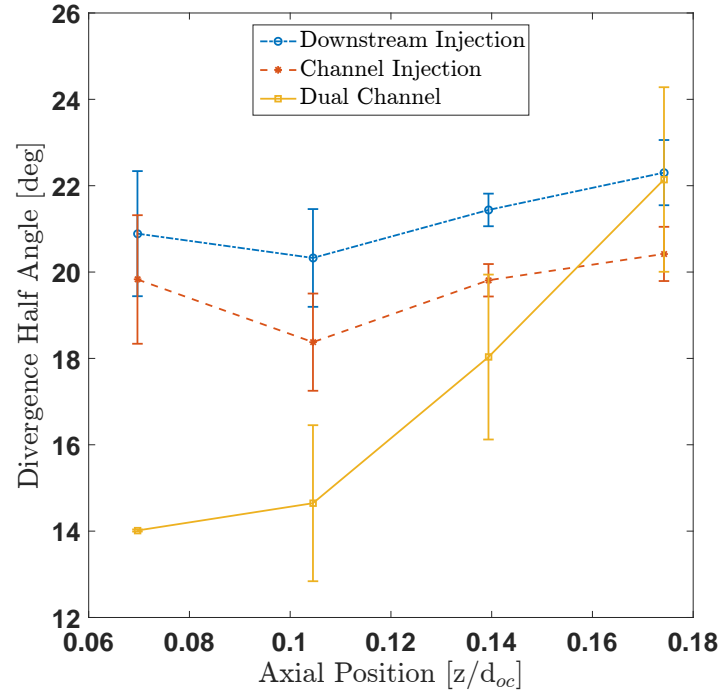

(a) Inner Channel divergence angle as a function of downstream position for the high-power cases.

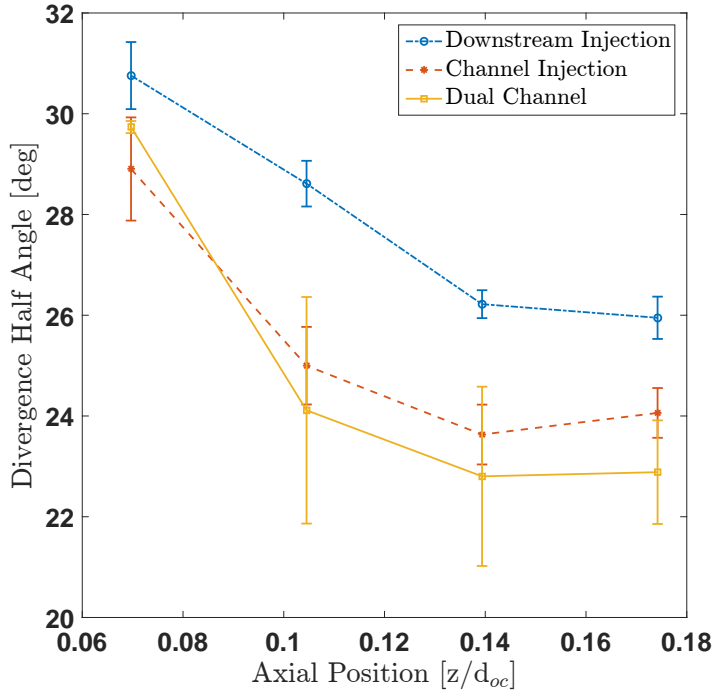

(b) Outer Channel divergence angle as a function of downstream position for the high-power cases.

Figure 10: Divergence angle as a function of downstream position for each high power test point. All axial positions are normalized by the outer channel diameter $\left(\mathrm{d}_{O C}\right)$. 
Figure 9, the radial location of the cutoff point for dual-channel mode is closer to the channel than in singlechannel mode. The peak for dual-channel mode is higher than the peak for a single channel, however the profiles follow each other closely far from the peak. It is important to note that the divergence angle when gas is injected via the other channel during the high-power case causes the outer channel divergence angle to decrease as seen in Figure 10b. This again indicates that raising the neutral density near the thruster exit plane leads to proper performance simulation of dual-channel mode.

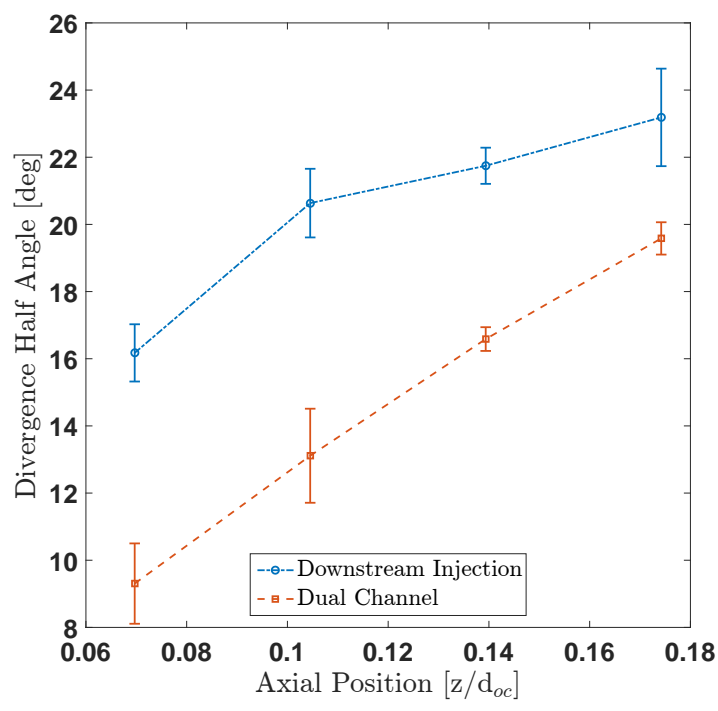

(a) Inner Channel divergence angle as a function of downstream position for the low-power cases.

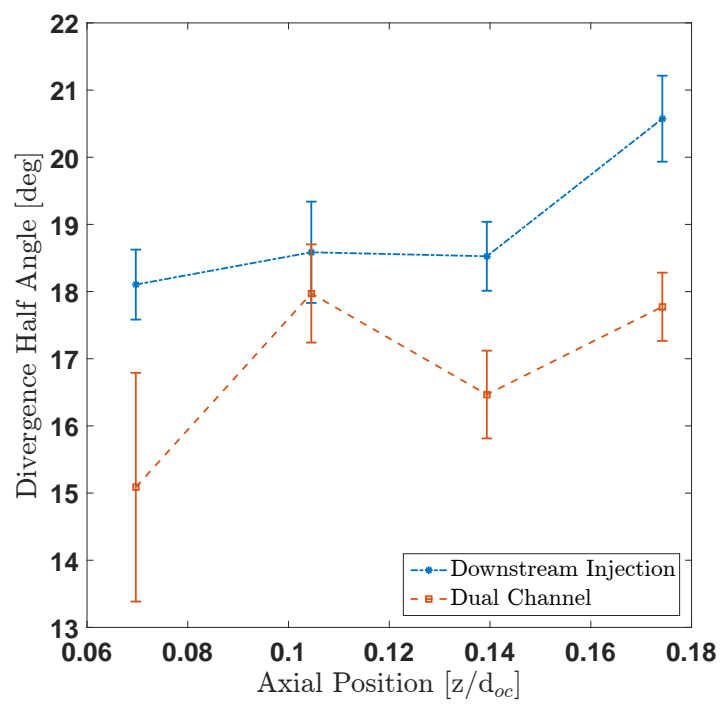

(b) Outer Channel divergence angle as a function of downstream position for the high-power cases.

Figure 11: Divergence angle as a function of downstream position for each low power test point. All axial positions are normalized by the outer channel diameter $\left(\mathrm{d}_{O C}\right)$.

In addition to divergence angle measurements, beam current was calculated. While a change in divergence angle suggests a change in acceleration region location, a change in beam current is more suggestive of neutral ingestion from the adjacent channel. Table 5 shows the results of beam current calculations. The

Table 5: Beam Current Values for High Power Operation

\begin{tabular}{c|c} 
Test Condition & Beam Current $[\mathrm{A}]$ \\
\hline \hline Inner Channel in Dual Channel & $7.90 \pm 0.10$ \\
\hline Inner Channel w/ Channel Injection & $7.74 \pm 0.04$ \\
\hline Inner Channel w/ Downstream Injection & $7.54 \pm 0.02$ \\
\hline \hline Outer Channel in Dual Channel & $20.45 \pm 0.30$ \\
\hline Outer Channel w/ Channel Injection & $20.77 \pm 0.01$ \\
\hline Outer Channel w/ Downstream Injection & $20.24 \pm 0.10$ \\
\hline
\end{tabular}

results for the inner channel show that dual-channel mode results in the highest beam current. Additionally, while not quite as high as dual channel mode, the beam current when gas is injection via the outer channel is higher than when gas is injected downstream. This suggests the inner channel is ingesting neutrals from the outer channel. Outer channel results are less clear as the uncertainty in dual channel mode is very high. However, it is clear that test point 8 yields higher beam currents than test point 7 suggesting that the outer channel will also ingest neutrals from the inner channel. The reason beam current in dual channel and single channel with channel injection modes do not match is reasonably explained via neutral flow path differences. When the channel is simply flowing cold gas, the neutrals exit the chamber in a diffuse pattern. However, 
the neutral flow path when there is plasma present is known to be quite different. ${ }^{18,19}$ This difference in flow pattern is the most likely cause for beam current differences.

\section{Conclusion}

Performance of a two-channel nested Hall thruster operating at $150 \mathrm{~V}$ anode-to-cathode potential was measured for two discharge power conditions. Thrust increased 5 and $11 \%$, for high power and low power respectively, in dual-channel mode versus the superposition of single channels when pressure was controlled via downstream injection. This difference was recovered when the pressure was controlled via injection through the non-operating channel for the high-power case. Channel injection was not used in the low power case, therefore it is unknown at this time, whether the effect holds across operating conditions. Pressure mapping shows that local thruster pressure in dual-channel mode is 1.6 times that of single-channel mode when chamber pressure is controlled with downstream injection. An effective anode efficiency and specific impulse show that the decreased divergence angle and increased beam current corresponds to an increase in thrust, anode efficiency, and anode specific impulse. The results indicate that at least part of the "anomalous" performance is due to neutral ingestion from the adjacent channel and the other part is due to divergence angle decreases. Further work will investigate this phenomena over a variety of operating conditions and thrusters. Additionally, a neutral flow model will be developed to calculate neutral density profiles near the thruster exit plane. Finally, it would be advantageous to be able to fire single channels on nested Hall thrusters and reproduce multi-channel operation performance and plasma properties. Therefore, further work will be done to determine whether both performance and plasma properties can be matched as only performance was matched in this study.

\section{Acknowledgments}

The authors would like to thank Dan Goebel for his help with the cathode and the entirety of the Plasmadynamics and Electric Propulsion Laboratory for their assistance and advice throughout the duration of the experiment. This work was supported by NASA Space Technology Research Fellowship grants NNX15AQ43H and NNX14AL65H.

\section{References}

1 "NASA Technology Roadmaps TA2: In-Space Propulsion Technologies," 2015.

${ }^{2}$ Florenz, R. E., The X3 100-kW Class Nested-Channel Hall Thruster: Motivation, Implementation and Initial Performance, Ph.D. thesis, University of Michigan, Ann Arbor, MI, 2014.

${ }^{3}$ Liang, R., The Combination of Two Concentric Discharge Channels into a Nested Hall-Effect Thruster, Ph.D. thesis, University of Michigan, Ann Arbor, MI, 2013.

${ }^{4}$ Hall, S. J., Cusson, S. E., and Gallimore, A. D., "30-kW Performance of a 100-kW Class Nested-channel Hall Thruster," 34th International Electric Propulsion Conference, Hyogo-Kobe, Japan, July 2015.

${ }^{5}$ Georgin, M.P., D. V. and Gallimore, A., "Investigation of Channel Interactions in a Nested Hall Thruster Part I: Acceleration Region Velocimetry," 52nd AIAA/SAE/ASEE Joint Propulsion Conference, Salt Lake City, Utah, July 2017.

-6Liang, R. and Gallimore, A. D., "Far-field Plume Measurements of a Nested-Channel Hall-Effect Thruster," 49th AIAA Aerospace Sciences Meeting including the New Horizons Forum and Aerospace Exposition, Orlando, Florida, January 2011.

${ }^{7}$ Liang, R. and Gallimore, A. D., "Constant-Power Performance and Plume Measurements of a Nested-Channel Hall-Effect Thruster," 32nd International Electric Propulsion Conference, Wiesbaden, Germany.

${ }^{8}$ Walker, M. L. and Gallimore, A. D., "Performance Characteristics of a Cluster of 5-kW Laboratory Hall Thrusters," Journal of Propulsion and Power, Vol. 23, No. 1, 2007, pp. 35-43.

${ }^{9}$ Polk, J. E., Pancotti, A., Haag, T., King, S., Walker, M., Blakely, J., and Ziemer, J., "Recommended Practices in Thrust Measurements," 33rd International Electric Propulsion Conference, Washington, D.C., October 2013.

${ }^{10}$ Hofer, R., Goebel, D. M., Mikellides, I. G., and Katz, I., "Design of a laboratory Hall Thruster with Magnetically Shielded Channel Walls, Phase II: Experiments," 48th AIAA/SAE/ASEE Joint Propulsion Conference, Atlanta, Georgia, 2012.

${ }^{11}$ Reid, B. M. and Gallimore, A. D., "Near-field Ion Current Density Measurements of a 6-kW Hall Thruster," 31st International Electric Propulsion Conference, Ann Arbor, MI, Ann Arbor, Michigan, 2009.

${ }^{12}$ Huang, W., Drenkow, B., and Gallimore, A. D., "Laser-Induced Fluorescence of Singly-Charged Xenon inside a 6-kW 
Hall Thruster," 45th AIAA/SAE/ASEE Joint Propulsion Conference, Denver, Colorado, August 2009.

${ }^{13}$ Randolph, T., Kim, V., Kaufman, H., Kozubsky, K., Zhurin, V. V., and Day, M., "Facility Effects on Stationary Plasma Thruster Testing," 23rd International Electric Propulsion Conference, Seattle, WA, September 1993.

${ }^{14}$ Hofer, R. R., Peterson, P. Y., and Gallimore, A. D., "Characterizing Vacuum Facility Backpressure Effects on the Performance of a Hall Thruster," 27th International Electric Propulsion Conference, Pasadena, CA, October 2001.

$\checkmark{ }^{15}$ Walker, M. L., Hofer, R. R., and Gallimore, A. D., "The Effects of Nude Faraday Probe Design and Vacuum Facility Backpressure on the Measured Ion Current Density Profile of Hall Thruster Plumes," 38th Joint Propulsion Conference, Indianapolis, Indiana, July 2002.

${ }^{16}$ Walker, M. L., Victor, A. L., Hofer, R. R., and Gallimore, A. D., "Effect of Backpressure on Ion Current Density Measurements in Hall Thruster Plumes," Journal of Propulsion and Power, Vol. 21, No. 3, 2005, pp. 408-415.

${ }^{17}$ Dankanich, J. W., Walker, M., Swiatek, M. W., and Yim, J. T., "Recommended Practice for Pressure Measurements and Calculation of Effective Pumping Speeds during Electric Propulsion Testing," 33rd International Electric Propulsion Conference, Washington D.C., USA, 2013.

${ }^{18}$ Huang, W., Gallimore, A. D., and Hofer, R. R., "Neutral Flow Evolution in a Six-Kilowatt Hall Thruster," Journal of Propulsion and Power, Vol. 27, No. 3, 2011, pp. 553-563.

${ }^{19}$ Reid, B. M. and Gallimore, A. D., "Review of Hall thruster neutral flow dynamics," 30th International Electric Propulsion Conference, Florence, Italy, September 2007. 
This article has been cited by:

1. Marcel P. Georgin, Vira Dhaliwal, Alec GallimoreInvestigation of Channel Interactions in a Nested Hall Thruster Part I: Acceleration Region Velocimetry . [Citation] [PDF] [PDF Plus] 